\title{
SUBALGEBRAS OF FREE RESTRICTED LIE ALGEBRAS
}

\author{
R.M. BRYANT, L.G. Kovács AND RALPh Stöhr
}

\begin{abstract}
A theorem independently due to A.I. Shirshov and E. Witt asserts that every subalgebra of a free Lie algebra (over a field) is free. The main step in Shirshov's proof is a little known but rather remarkable result: if a set of homogeneous elements in a free Lie algebra has the property that no element of it is contained in the subalgebra generated by the other elements, then this subset is a free generating set for the subalgebra it generates. Witt also proved that every subalgebra of a free restricted Lie algebra is free. Later G.P. Kukin gave a proof of this theorem in which he adapted Shirshov's argument. The main step is similar, but it has come to light that its proof contains substantial gaps. Here we give a corrected proof of this main step in order to justify its applications elsewhere.
\end{abstract}

\section{INTRODUCTION}

The Shirshov-Witt Theorem $[\mathbf{9}, \mathbf{1 1}]$ asserts that every subalgebra of a free Lie algebra (over a field) is free. Witt [11] also gave a similar result for restricted Lie algebras (over fields of positive characteristic), and of course there are some even better known results of the same kind in other branches of algebra, particularly for free groups and free Abelian groups. Shirshov's proof in [9] started with an easy application of a method of Kurosh to show that each subalgebra of a free Lie algebra has a generating set $S$ which is reduced in the following sense: for each $s \in S$ the leading term of $s$ (that is, the highest degree homogeneous component of $s$ ) does not belong to the subalgebra generated by the leading terms of the other elements of $S$. The second and main step of the proof was to show that every reduced subset of a free Lie algebra is independent in the sense that it is a free generating set for the subalgebra it generates. This step was not proclaimed as a lemma or theorem in its own right and has not become well-known like the Shirshov-Witt Theorem, but it is a remarkable result with, as far as we are aware, no non-trivial parallels in other branches of algebra. It may have contributed to this lack of recognition that [9] is still only available in the original Russian and that books presenting the Shirshov-Witt Theorem have chosen proofs which do not involve this step.

One might try to modify the definition of a reduced set to say that a set $S$ is irredundant if no element of $S$ belongs to the subalgebra generated by the other elements.

Received 18th April, 2005

Copyright Clearance Centre, Inc. Serial-fee code: 0004-9727/05 \$A2.00+0.00. 
Shirshov's main step yields that every irredundant set of homogeneous elements is independent. However, the homogeneity condition cannot be removed. For example, in the Lie algebra $L$ freely generated by $x$ and $y$, let

$$
S=\{x, x+[y, x], y+[[y, x], x]\}
$$

Then it is not difficult to verify that $S$ is irredundant; but $S$ is not independent (because $S$ generates $L$ ). It should also be noted that independent sets need not be reduced: consider, for example, the set $\{x, y, z+[y, x]\}$ in the Lie algebra freely generated by $x$, $y$ and $z$.

Kukin [7] adapted Shirshov's argument to give a proof of Witt's result for free restricted Lie algebras. This adaptation contained an analogue of Shirshov's main step, this time proclaimed as a lemma. With definitions of reduced and independent as before, except that subalgebra now means restricted Lie subalgebra, Kukin's Lemma (Lemma 2 in [7]) states that every reduced subset of a free restricted Lie algebra is independent.

In that paper, and later also in Bakhturin's book [1], this result was used not only in the proof of Witt's Theorem, but (for instance) also in proving Kukin's formula for the free rank of a subalgebra of finite codimension in a free restricted Lie algebra (the analogue of Schreier's formula so well known in group theory). We have found it an indispensable tool in $[2,3,6]$, and have little doubt that it will find many further applications.

However, we have been aware for some time that both of its proofs in print, the original in [7] and the one given in [1], contain substantial gaps (see the Remarks in the last section of this note). Our confidence in the result was sustained by possible alternative approaches, but recently we found that Kukin's proof may be corrected by extending and modifying his argument. The main purpose of this note is to provide firm foundations for the applications of the result by making available our corrected proof. A consolidated exposition seems preferable to a list of corrigenda, and then it is only one short step to complete Kukin's proof of Witt's Theorem: we include that to make the readers' labour more rewarding.

The focus here is on free restricted Lie algebras, but only minor modifications are needed to deal with the case of free Lie algebras, as considered by Shirshov. We hope that our exposition will bring wider attention to the powerful and rather striking results of Shirshov and Kukin that have been described above.

\section{Free RESTRICTED LiE ALgebras}

Let $K$ be a field of prime characteristic $p$. When considering Lie algebras over $K$ we write the Lie product of elements $u$ and $v$ as $[u, v]$, and $\left[u_{1}, u_{2}, \ldots, u_{n}\right]$ denotes the left-normed product $\left[\cdots\left[\left[u_{1}, u_{2}\right], \ldots\right], u_{n}\right]$.

A restricted Lie algebra $R$ over $K$ is a Lie algebra over $K$ with an additional unary " $p$-powering" operation, in which the image of each element $u$ of $R$ is written as a formal 
power $u^{p}$. The properties of this operation are $(\alpha u)^{p}=\alpha^{p} u^{p}$ for all $\alpha \in K, u \in R$, where $a^{p}$ denotes the usual $p$ th power,

$$
\left[u, v^{p}\right]=[u, \underbrace{v, \ldots, v}_{p}]
$$

for all $u, v \in R$, and $(u+v)^{p}=u^{p}+v^{p}+l(u, v)$, for all $u, v \in R$, where $l(u, v)$ is a certain linear combination of Lie products (see [5, Definition V.4] or [8, Section 2.5.2] for the precise form).

Free restricted Lie algebras may be defined by means of a universal property. However, they arise in a concrete way from free associative algebras, as now described. For many purposes this is the easiest way of thinking about them.

Let $A$ be a free associative algebra over $K$ with a free generating set $X$. Then $A$ may be regarded as a restricted Lie algebra with Lie multiplication given by $[u, v]=u v-v u$ and $p$-powering given by

$$
u \mapsto u^{p}=\underbrace{u \cdots u}_{p} .
$$

The restricted Lie subalgebra $R$ of $A$ generated by $X$ is a free restricted Lie algebra with free generating set $X$ (see, for example, [8, Section 5.2]). If $|X|=r$ then $R$ is said to have rank $r$.

For each positive integer $n$ let $A_{n}$ be the subspace of $A$ spanned by all products of the form $u_{1} u_{2} \cdots u_{n}$ with $u_{1}, \ldots, u_{n} \in X$, and write $R_{n}=R \cap A_{n}$. It is easily proved that $R_{n}$ is spanned by all monomials that have the form $u^{p^{k}}$ where $k \geqslant 0, p^{k} \mid n$, and $u$ is the Lie product, with some bracketing, of $n / p^{k}$ not necessarily distinct elements of $X$.

As a vector space, $R$ has the decomposition $R=\bigoplus_{n} R_{n}$. Furthermore, $R$ is graded as an algebra: if $u \in R_{m}$ and $v \in R_{n}$ then $[u, v] \in R_{m+n}$ and $u^{p} \in R_{p m}$. An element $u$ of $R$ is said to be homogeneous if $u \in R_{n}$ for some $n$. Each element $u$ of $R$ may be written uniquely in the form $u=\sum_{n} u_{n}$, where $u_{n} \in R_{n}$ for all $n$ and only finitely many of the $u_{n}$ are non-zero. If $u \neq 0$ we write $\operatorname{deg} u$ for the largest value of $n$ for which $u_{n} \neq 0$. This is called the degree of $u$. The corresponding term $u_{n}$ is called the leading term of $u$ and denoted by $\bar{u}$. For $u=0$ we define $\operatorname{deg} u=0$ and $\bar{u}=0$. Furthermore, if $S$ is a finite subset of $R$ we write

$$
\operatorname{deg} S=\sum_{u \in S} \operatorname{deg} u .
$$

This is called the degree sum of $S$.

By a subalgebra of $R$ we always mean a restricted Lie subalgebra, and the subalgebra generated by a set $S$ is denoted by $\langle S\rangle$. A subset $S$ is said to be independent if $S$ is a free generating set for $\langle S\rangle$, and $S$ is said to be reduced if, for all $u \in S$,

$$
\bar{u} \notin\langle\bar{w}: w \in S \backslash\{u\}\rangle .
$$


The concepts of homogeneous element, degree, leading term and reduced subset are defined in terms of the grading of $R$ given by the free generating set $X$. Unless otherwise specified this grading is taken as fixed.

In [7], Kukin used the alternative terminology of Lie $p$-algebras, instead of restricted Lie algebras. He referred to $p$-subalgebras, $p$-reduced subsets and $p$-independent subsets, presumably in order to avoid any confusion with the corresponding concepts for Lie algebras discussed in Shirshov's paper [9]. Since we concentrate on restricted Lie algebras there should be no confusion here.

Then [7, Lemma 4] may be paraphrased as follows.

LEMMA 2.1. Let $R$ be a free restricted Lie algebra with free generating set $\left\{x_{1}, \ldots, x_{r}\right\}$, where $r$ is a positive integer. Then the ideal $I$ of $R$ generated by $x_{1}^{p}, x_{2}, \ldots, x_{r}$ is a free subalgebra of $R$ with a free generating set $Y$ consisting of $x_{1}^{p}$ and the elements

$$
[x_{i}, \underbrace{x_{1}, \ldots, x_{1}}_{c}]
$$

for $i=2, \ldots, r$ and $c=0, \ldots, p-1$.

We have no reservations about the proof of this lemma in [7] or in Bakhturin's book [1, Section 2.7, proof of Witt's Theorem], so we give no proof here. This lemma, in the terminology of [10], is called "restricted elimination". There was a corresponding result for free Lie algebras in [9] and that was the simplest special case of what has since become known as "elimination" or "Lazard elimination" (see, for example, [4, Proposition 10 in Section 2.9, Chapter 2] or [8, Section 0.3]).

The subalgebra $I$ of $R$ described in Lemma 2.1 has a grading determined by its free generating set $Y$. For elements and subsets of $I$ it is sometimes necessary to distinguish between the concepts homogeneous, degree, leading term and reduced defined in terms of $X$ and the same concepts defined in terms of $Y$. When necessary we make this explicit. For example, we write $\operatorname{deg}_{X} u$ and $\operatorname{deg}_{Y} u$ for the degrees of an element $u$ of $I$ with respect to $X$ and $Y$, respectively.

Our main object here is to give a corrected proof of [7, Lemma 2], namely the following result.

Lemma 2.2. (Kukin's Lemma) Let $S$ be a reduced subset of a free restricted Lie algebra $R$. Then $S$ is independent.

Once the machinery for the proof of Lemma 2.2 has been set up it is rather easy to prove the following result, [7, Lemma 1$]$.

LEMma 2.3. Let $Q$ be a subalgebra of a free restricted Lie algebra $R$. Then $Q$ has a reduced generating set.

We include a proof of this result partly because Kukin suppressed some of the details but mainly because Lemmas 2.2 and 2.3 immediately give Witt's Theorem. 
THEOREM 2.4. (Witt [11]) Let $Q$ be a subalgebra of a free restricted Lie algebra $R$. Then $Q$ is free.

\section{Preliminary Results}

Throughout this section we take $K$ to be a field of prime characteristic $p$ and $R$ to be a free restricted Lie algebra over $K$.

Let $\phi$ or $\phi\left(y_{1}, \ldots, y_{m}\right)$ be a monomial in a free restricted Lie algebra over $K$ with free generating set $\left\{y_{1}, \ldots, y_{m}\right\}$ and let $u_{1}, \ldots, u_{m}$ be homogeneous elements of $R$. Then either $\phi\left(u_{1}, \ldots, u_{m}\right)=0$ or $\phi\left(u_{1}, \ldots, u_{m}\right)$ has degree $\sum_{j} d_{j} \operatorname{deg} u_{j}$, where $d_{j}$ is the degree of $y_{j}$ in $\phi$. We call the number $\sum_{j} d_{j} \operatorname{deg} u_{j}$ the formal degree of $\phi\left(u_{1}, \ldots, u_{m}\right)$ and denote it by $\operatorname{Deg} \phi\left(u_{1}, \ldots, u_{m}\right)$.

Lemma 3.1. Let $S$ be a finite subset of $R$ which is not independent. If the leading terms of distinct elements of $S$ are distinct then the set of these leading terms is not independent.

Proof: Let $S=\left\{s_{1}, \ldots, s_{m}\right\}$. Clearly we may assume that each $s_{i}$ is non-zero. Since $S$ is not independent there exists a non-zero element $\phi$ in a free restricted Lie algebra of rank $m$ such that $\phi\left(s_{1}, \ldots, s_{m}\right)=0$. We can write $\phi=\sum_{j=1}^{l} \alpha_{j} \phi_{j}$ where the $\alpha_{j}$ are non-zero elements of $K$ and the $\phi_{j}$ are linearly independent monomials.

Let $n$ be the maximum of the formal degrees $\operatorname{Deg} \phi_{j}\left(\bar{s}_{1}, \ldots, \bar{s}_{m}\right)$. We can renumber the $\phi_{j}$ so that, for some $k>0, \operatorname{Deg} \phi_{j}\left(\bar{s}_{1}, \ldots, \bar{s}_{m}\right)=n$ for $j \leqslant k$ and $\operatorname{Deg} \phi_{j}\left(\bar{s}_{1}, \ldots, \bar{s}_{m}\right)$ $<n$ for $j>k$.

For $j \leqslant k$ we have

$$
\phi_{j}\left(s_{1}, \ldots, s_{m}\right)=\phi_{j}\left(\bar{s}_{1}, \ldots, \bar{s}_{m}\right)+v_{j}
$$

where $\operatorname{deg} v_{j}<n$. Also, for $j>k$, we have $\operatorname{deg} \phi_{j}\left(s_{1}, \ldots, s_{m}\right)<n$. Write $\phi^{*}=\sum_{j=1}^{k} \alpha_{j} \phi_{j}$. Then $\phi^{*} \neq 0$ and

$$
0=\phi\left(s_{1}, \ldots, s_{m}\right)=\phi^{*}\left(\bar{s}_{1}, \ldots, \bar{s}_{m}\right)+v
$$

where $\phi^{*}\left(\bar{s}_{1}, \ldots, \bar{s}_{m}\right) \in R_{n}$ and $\operatorname{deg} v<n$. Thus $\phi^{*}\left(\bar{s}_{1}, \ldots, \bar{s}_{m}\right)=0$. Hence $\left\{\bar{s}_{1}, \ldots, \bar{s}_{m}\right\}$ is not independent.

LEMMA 3.2. Let $S$ be a finite set of non-zero elements of $R$ and suppose that $S$ is not reduced. Then, for some $u \in S$, there exists $w \in\langle S \backslash\{u\}\rangle$ such that $\operatorname{deg}(u-w)$ $<\operatorname{deg} u$. Furthermore we may take $w$ in the form $w=w_{1}+w_{2}$, where $w_{1}$ is a linear combination of elements of $S \backslash\{u\}$ of degree equal to $\operatorname{deg} u$ and $w_{2}$ belongs to the subalgebra of $R$ generated by the elements of $S \backslash\{u\}$ of degree smaller than $\operatorname{deg} u$. 
ProOF: By assumption there exists $u \in S$ such that

$$
\bar{u} \in\langle\bar{v}: v \in S \backslash\{u\}\rangle
$$

Write $S \backslash\{u\}=\left\{s_{1}, \ldots, s_{m}\right\}$. There exists an element $\phi$ in a free restricted Lie algebra of rank $m$ such that $\bar{u}=\phi\left(\bar{s}_{1}, \ldots, \bar{s}_{m}\right)$. Write $\phi=\sum_{j=1}^{l} \alpha_{j} \phi_{j}$ where the $\alpha_{j}$ belong to $K$ and the $\phi_{j}$ are monomials. Let $n=\operatorname{deg} u=\operatorname{deg} \bar{u}$. We consider the formal degrees $\operatorname{Deg} \phi_{j}\left(\bar{s}_{1}, \ldots, \bar{s}_{m}\right)$ and renumber the $\phi_{j}$ so that, for some $k, \operatorname{Deg} \phi_{j}\left(\bar{s}_{1}, \ldots, \bar{s}_{m}\right)=n$ for $j \leqslant k$ and $\operatorname{Deg} \phi_{j}\left(\bar{s}_{1}, \ldots, \bar{s}_{m}\right) \neq n$ for $j>k$. Write $\phi^{*}=\sum_{j=1}^{k} \alpha_{j} \phi_{j}$. Then $\bar{u}=\phi^{*}\left(\bar{s}_{1}, \ldots, \bar{s}_{m}\right)$. Let $w=\phi^{*}\left(s_{1}, \ldots, s_{m}\right)$. For $j=1, \ldots, k$ we have

$$
\phi_{j}\left(s_{1}, \ldots, s_{m}\right)=\phi_{j}\left(\bar{s}_{1}, \ldots, \bar{s}_{m}\right)+v_{j}
$$

where $\operatorname{deg} v_{j}<n$. Thus

$$
w=\phi^{*}\left(s_{1}, \ldots, s_{m}\right)=\phi^{*}\left(\bar{s}_{1}, \ldots, \bar{s}_{m}\right)+v=\bar{u}+v
$$

where $\operatorname{deg} v<n$. Hence $\operatorname{deg}(u-w)<n$.

We may renumber $\phi_{1}, \ldots, \phi_{k}$ so that, for some $t, \operatorname{deg} \phi_{j}=1$ for $j \leqslant t$ and $\operatorname{deg} \phi_{j}>1$ for $j>t$. Then $w=w_{1}+w_{2}$ where

$$
w_{1}=\sum_{j=1}^{t} \alpha_{j} \phi_{j}\left(s_{1}, \ldots, s_{m}\right) \quad \text { and } \quad w_{2}=\sum_{j=t+1}^{k} \alpha_{j} \phi_{j}\left(s_{1}, \ldots, s_{m}\right)
$$

Since $\operatorname{Deg} \phi_{j}\left(\bar{s}_{1}, \ldots, \bar{s}_{m}\right)=n$ for $j=1, \ldots, k$, the elements $w_{1}$ and $w_{2}$ have the required properties.

LEMMA 3.3. Let $S$ be a reduced set of homogeneous elements of $R$. Then no set of cardinality smaller than $|S|$ can generate $\langle S\rangle$.

Proof: Let $Q=\langle S\rangle$. Write $Q^{\prime}$ for the ideal of $Q$ generated by all elements of the forms $u^{p}$ and $[u, v]$ where $u, v \in Q$. Then any generating set of the restricted Lie algebra $Q / Q^{\prime}$ spans it as a vector space. If $W$ is any generating set of $Q$ it follows that $W$ spans $Q$ modulo $Q^{\prime}$. Hence $|W| \geqslant \operatorname{dim} Q / Q^{\prime}$. Thus it suffices to show that the elements of $S$ are linearly independent modulo $Q^{\prime}$.

Suppose that this is not the case. Then for some $u \in S$ there exist elements $u_{1}, \ldots, u_{m} \in S \backslash\{u\}, \alpha_{1}, \ldots, \alpha_{m} \in K$ and $v \in Q^{\prime}$ such that

$$
u=v+\sum_{j=1}^{m} \alpha_{j} u_{j}
$$

Let $\Omega$ be the subset of $Q$ consisting of the elements $s^{\mathrm{p}^{k}}$ where $s \in S$ and $k \geqslant 1$ and the elements $w^{p^{k}}$ where $w$ is a Lie product (with arbitrary bracket arrangement) of two 
or more (not necessarily distinct) elements of $S$ and $k \geqslant 0$. Thus each element of $\Omega$ is homogeneous. It is straightforward to verify that $Q^{\prime}$ is spanned by $\Omega$. Thus $v$ is a linear combination of elements of $\Omega$.

Write $n=\operatorname{deg} u$. We may renumber $u_{1}, \ldots, u_{m}$ so that, for some $k \operatorname{deg} u_{j}=n$ for $j \leqslant k$ and $\operatorname{deg} u_{j} \neq n$ for $j>k$. Then, by comparing terms of degree $n$ in the equation $u=v+\sum \alpha_{j} u_{j}$ we obtain a relation

$$
u=v^{\prime}+\sum_{j=1}^{k} \alpha_{j} u_{j}
$$

where $v^{\prime}$ is a linear combination of elements of $\Omega$ of degree $n$.

By the definition of $\Omega$, every element of $\Omega$ of degree $n$ is formed from elements of $S$ of degree smaller than $n$. Thus $v^{\prime} \in\langle S \backslash\{u\}\rangle$ and we obtain $u \in\langle S \backslash\{u\}\rangle$. This contradicts the fact that $S$ is reduced and completes the proof.

Lemma 3.4. Let $S$ be a subset of $R$. Let $u \in S$ and $w \in\langle S \backslash\{u\}\rangle$, and write $S^{*}=(S \backslash\{u\}) \cup\{u-w\}$. If $u-w \notin S \backslash\{u\}$ and $S^{*}$ is independent then $S$ is independent.

Proof: Clearly $\left\langle S^{*}\right\rangle=\langle S\rangle$. Suppose that $u-w \notin S \backslash\{u\}$ and $S^{*}$ is independent. Then there are endomorphisms $\sigma$ and $\tau$ of $\left\langle S^{*}\right\rangle$ given by

$$
\begin{array}{ll}
s \sigma=s \text { for } s \in S \backslash\{u\}, & (u-w) \sigma=u, \\
s \tau=s \text { for } s \in S \backslash\{u\}, & (u-w) \tau=u-2 w .
\end{array}
$$

It is easily proved that $\sigma$ and $\tau$ are mutually inverse. Thus $\sigma$ is an automorphism. Since $S=S^{*} \sigma$, it follows that $S$ is independent.

\section{KUKIN'S PROOF OF WiTT'S THEOREM}

In this section we prove Lemmas 2.2 and 2.3. As already remarked, they immediately yield Witt's Theorem, Theorem 2.4. Since the main purpose of our work is the correction of Kukin's proof in [7] and the account of it in [1] we follow the proof of Lemma 2.2 with a brief indication of the problems with the original proofs.

Proof of LemMa 2.2: It is clearly sufficient to prove the result in the case where $S$ is finite and $R$ has finite rank. We assume that there is a counterexample, that is, a pair $(S, R)$ where $S$ is a finite and reduced, but not independent, subset of $R$, and $R$ has finite rank. We shall obtain a contradiction.

We choose $n$ as small as possible such that there exists a counterexample $(S, R)$ where $S$ has degree sum $n$, that is, $\operatorname{deg} S=n$. We then choose $r$ as small as possible such that there exists a counterexample $(S, R)$ with $\operatorname{deg} S=n$ and $R$ of rank $r$. Let $X$ be a free generating set of $R$, where $X=\left\{x_{1}, \ldots, x_{r}\right\}$. 
Since $S$ is reduced, distinct elements of $S$ have distinct leading terms. Let $H$ be the set of these leading terms. Note that the elements of $H$ are non-zero and homogeneous and $H$ is reduced. By Lemma 3.1, $H$ is not independent. Also, $\operatorname{deg} H=n$.

Suppose that the elements of $H$ of degree 1 are $z_{1}, \ldots, z_{s}$. Since $H$ is reduced, $\left\{z_{1}, \ldots, z_{s}\right\}$ is a linearly independent subset of $R_{1}$. If $s=r$ then $\left\{z_{1}, \ldots, z_{s}\right\}$ is a free generating set for $R$ and, since $H$ is reduced, we get $H=\left\{z_{1}, \ldots, z_{s}\right\}$, contrary to the fact that $H$ is not independent. Hence $s<r$. It follows that there is an automorphism $\theta$ of $R$ which maps $R_{1}$ to $R_{1}$ and maps $\left\{z_{1}, \ldots, z_{s}\right\}$ to a subset of $\left\{x_{2}, \ldots, x_{r}\right\}$. Then $H \theta$ has all the properties of $H$. Thus without loss of generality we may assume that the elements of $H$ of degree 1 all belong to $\left\{x_{2}, \ldots, x_{r}\right\}$.

Let $I$ be the ideal of $R$ generated by $x_{1}^{p}, x_{2}, \ldots, x_{r}$. All monomials of $R$ of degree greater than 1 belong to $I$. Therefore $H \subseteq I$. Let $Y$ be the free generating set for $I$ given by Lemma 2.1. When the elements of $H$ are written with respect to $Y$ they need not be homogeneous. However, for $h \in H$, we may write $h=h_{1}+h_{2}$ where $h_{1} \in\left\langle x_{2}, \ldots, x_{r}\right\rangle$ and either $h_{1}=0$ or $\operatorname{deg}_{Y} h_{1}=\operatorname{deg}_{X} h$ and where $\operatorname{deg}_{Y} h_{2}<\operatorname{deg}_{X} h$. Thus $\operatorname{deg}_{Y} H \leqslant \operatorname{deg}_{X} H=n$ and if $\operatorname{deg}_{Y} H=\operatorname{deg}_{X} H$ then the leading terms of the elements of $H$ with respect to $Y$ all belong to $\left\langle x_{2}, \ldots, x_{r}\right\rangle$.

The definition of independence for a set involves only the subalgebra it generates. Hence $H$ is not independent when considered with respect to $Y$.

Suppose that $H$ is reduced with respect to $Y$. Then the minimality of $n$ gives $\operatorname{deg}_{Y} H=n$. Since $H$ is reduced, the leading terms of distinct elements of $H$ are distinct. Let $\bar{H}$ be the set of these. Clearly $\bar{H}$ is reduced (with respect to $Y$ ) and $\operatorname{deg}_{Y} \bar{H}=n$. By Lemma $3.1, \bar{H}$ is not independent. However, as observed above, $\bar{H} \subseteq\left\langle x_{2}, \ldots, x_{r}\right\rangle$. This contradicts the minimality of $r$. Hence $H$ is not reduced with respect to $Y$.

Consider the subsets $N$ of $\langle H\rangle$ satisfying $\langle N\rangle=\langle H\rangle,|N|=|H|, 0 \notin N, N$ is not independent, $N$ is not reduced with respect to $Y$, and $\operatorname{deg}_{Y} N \leqslant n$. For example, we can take $N=H$. Among these sets, choose $N$ so that $\operatorname{deg}_{Y} N$ is as small as possible. By Lemma 3.2, there exist $u \in N$ and $w \in\langle N \backslash\{u\}\rangle$ such that $\operatorname{deg}_{Y}(u-w)<\operatorname{deg}_{Y} u$. Write $N^{*}=(N \backslash\{u\}) \cup\{u-w\}$. Clearly $\left\langle N^{*}\right\rangle=\langle N\rangle=\langle H\rangle$. Since $H$ is reduced and consists of homogeneous elements with respect to $X$, it follows by Lemma 3.3 that $u-w \notin\langle N \backslash\{u\}\rangle$. Thus $\left|N^{*}\right|=|N|=|H|$ and the elements of $N^{*}$ are non-zero. Also, by Lemma $3.4, N^{*}$ is not independent. Clearly $\operatorname{deg}_{Y} N^{*}<\operatorname{deg}_{Y} N \leqslant n$. Thus, by the minimality of $n, N^{*}$ is not reduced. This contradicts the choice of $N$ and completes the proof of the lemma. $]$

REMARKS 1. The last two paragraphs of our proof of Lemma 2.2 should be compared with the last paragraph of Kukin's proof of his Lemma 2. In terms of the notation used here, Kukin claims that $\operatorname{deg}_{Y} H<\operatorname{deg}_{X} H$. This does not hold, for example, if $H$ is the set

$$
\left\{x_{2},\left[x_{3}, x_{2}\right]+\left[x_{2}, x_{1}\right]\right\} \text {. }
$$

We have had to treat the case $\operatorname{deg}_{Y} H=\operatorname{deg}_{X} H$ by observing that $\bar{H} \subseteq\left\langle x_{2}, \ldots, x_{r}\right\rangle$ 
and using Lemma 3.1. Kukin's use of "elementary transformations" is essentially the passage from $N$ to $N^{*}$ in our proof. However, Kukin does not consider the possibility that $u-w=0$. To show that this case does not arise we have had to introduce Lemma 3.3.

In the version of the proof given in [1, Section 2.7, proof of Witt's Theorem] it seems that the assumption is made that $H$ remains homogeneous and reduced when written with respect to $Y$. However, neither property is preserved, for example, if $p>3$ and $H$ is the set

$$
\left\{\left[\left[x_{2}, x_{1}\right],\left[x_{3}, x_{1}\right]\right]+\left[x_{2}, x_{1}, x_{1}, x_{1}\right],\left[x_{2}, x_{1}\right],\left[x_{3}, x_{1}\right]\right\} .
$$

PROOF OF LEMMA 2.3: For each non-negative integer $n$, let $Q_{n}$ be the subspace of $Q$ consisting of all elements $u$ of $Q$ such that $\operatorname{deg} u \leqslant n$. (In particular, $Q_{0}=\{0\}$.) For each $n \geqslant 1$, let $V_{n}$ be a subspace of $Q_{n}$ such that

$$
Q_{n}=\left(\left(Q_{n-1}\right\rangle \cap Q_{n}\right) \oplus V_{n}
$$

and let $S_{n}$ be a basis of $V_{n}$. Define $S=\bigcup_{n} S_{n}$. It follows by induction that $\left\langle Q_{n}\right\rangle$ $=\left\langle S_{1} \cup \cdots \cup S_{n}\right\rangle$ for all $n$. Hence $Q=\langle S\rangle$. It remains to prove that $S$ is reduced.

Suppose otherwise. Note that, by construction, every element of $S$ is non-zero. Since $S$ is not reduced it contains a finite subset which is not reduced. Thus we can apply Lemma 3.2. There exist $u \in S$ and $w \in\langle S \backslash\{u\}\rangle$ such that $\operatorname{deg}(u-w)<\operatorname{deg} u$ and $w=w_{1}+w_{2}$, where $w_{1}$ and $w_{2}$ are as in Lemma 3.2. Let $n=\operatorname{deg} u$. Thus $u \in S_{n}, w_{1}$ is a linear combination of elements of $S_{n} \backslash\{u\}$ and $w_{2} \in\left\langle Q_{n-1}\right\rangle$. By the choice of $S_{n}, u-w_{1}$ is a non-zero element of $V_{n}$. However, $\operatorname{since} \operatorname{deg}(u-w)<n$, we have $u-w \in Q_{n-1}$. Hence

$$
u-w_{1}=(u-w)+w_{2} \in\left\langle Q_{n-1}\right\rangle .
$$

Thus $u-w_{1}$ is a non-zero element of $\left\langle Q_{n-1}\right\rangle \cap V_{n}$. However,

$$
\left\langle Q_{n-1}\right\rangle \cap V_{n}=\left(\left\langle Q_{n-1}\right\rangle \cap Q_{n}\right) \cap V_{n}=\{0\} .
$$

This is a contradiction, completing the proof of the lemma.

\section{REFERENCES}

[1] Yu. A. Bakhturin, Identical relations in Lie algebras, (Russian) (Nauka, Moscow, 1985). English translation: (VNU Science Press, Utrecht, 1987).

[2] R.M. Bryant and R. Stöhr, 'On the module structure of free Lie algebras', Trans. Amer. Math. Soc. 352 (2000), 901-934.

[3] R.M. Bryant, L.G. Kovács and R. Stöhr, 'Lie powers of modules for groups of prime order', Proc. London Math. Soc. (3) 84 (2002), 343-374.

[4] N. Bourbaki, Lie groups and Lie algebras, (Part I: Chapters 1-3) (Hermann, Paris, 1975).

[5] N. Jacobson, Lie algebras (Interscience, New York, 1962). 
[6] L.G. Kovács and R. Stöhr, 'Lie powers of the natural module for $G L(2)$ ', J. Algebra 229 (2000), 435-462.

[7] G.P. Kukin, 'Subalgebras of free Lie p-algebras', (Russian), Algebra i Logika 11 (1972), 535-550. English translation: Algebra Logic 11 (1972), 294-303.

[8] C. Reutenauer, Free Lie algebras (Clarendon Press, Oxford, 1993).

[9] A.I. Shirshov, 'Subalgebras of free Lie algebras', (Russian), Mat. Sbornik N. S. 33 (1953), 441-452.

[10] R. Stöhr, 'Restricted Lazard elimination and modular Lie powers', J. Austral. Math. Soc. 71 (2001), 259-277.

[11] E. Witt, 'Die Unterringe der freien Lieschen Ringe', Math. Z. 64 (1956), 195-216.

School of Mathematics

University of Manchester

PO Box 88

Manchester M60 1QD

United Kingdom

e-mail: roger.bryant@manchester.ac.uk ralph.stohr@manchester.ac.uk
Australian National University

Canberra ACT 0200

Australia

e-mail: kovacs@maths.anu.edu.au 